\section{Stent migration into the peritoneal cavity following endoscopic ultrasound-guided hepaticogastrostomy}

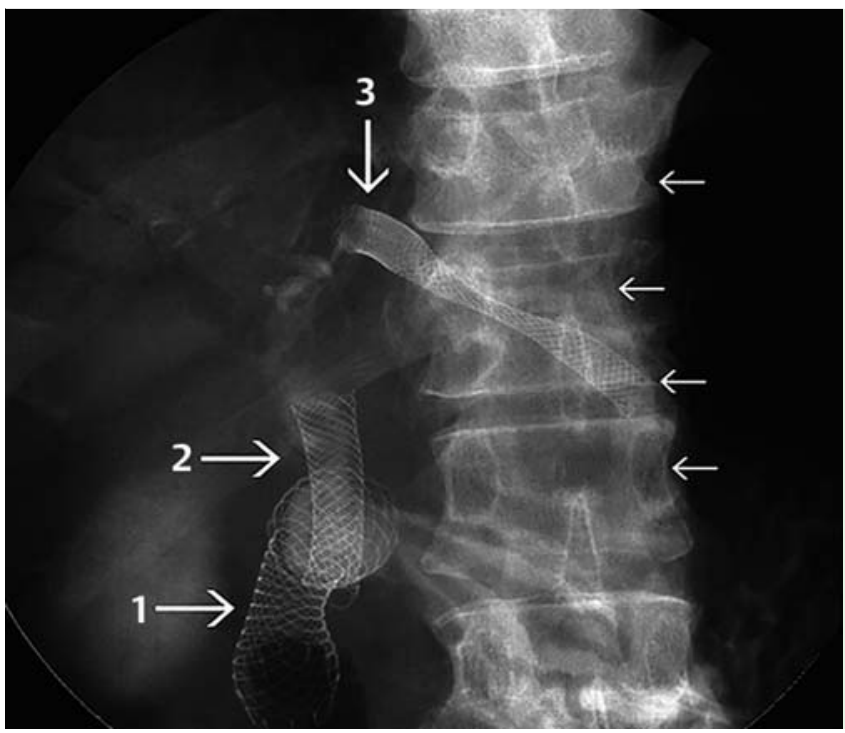

Fig. 1 Stent migration following endoscopic ultrasound (EUS)-guided hepaticogastrostomy in a 54-year-old man with unresectable pancreatic cancer. The proximal end of the stent is located outside the gastric wall. 1, duodenal stent, 2, choledochoduodenostomy stent, 3 , hepaticogastrostomy stent; small arrows, gastric wall.

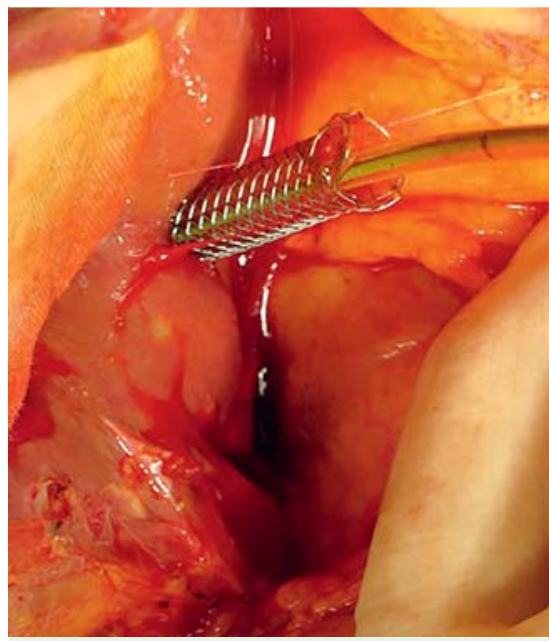

Fig. 2 Endoscopic ultrasound-guided hepaticogastrostomy was attempted for biliary drainage in a 54-year-old man with unresectable pancreatic cancer. The stent immediately migrated into the peritoneal cavity; drainage was therefore done by open surgery. The migrated proximal end of the stent can be seen in the peritoneal cavity.

It is not always possible to perform endoscopic retrograde cholangiopancreatography, and endoscopic ultrasound-guided biliary drainage (EUS-BD) has been performed as an alternative to percutaneous or surgical approaches [1,2]. The 2008 EUS Working Group summarized the indications, techniques, and complications of EUS-guided hepaticogastrostomy

trol 19G; Medi-Globe GmbH, Rosenheim, later, he died as a result of peritonitis carcinomatosa.

Anatomically, the stomach is not directly attached to the liver, and during EUS-HGS there is no space between these organs when the echoendoscope is pressed against the stomach wall. Pulling back the echoendoscope for stent placement creates space between the liver and stomach wall. Given this complication, stents with lengths of 10 or $12 \mathrm{~cm}$ should be used to avoid inward stent migration.

Endoscopy_UCTN_Code_CPL_1AK_2AD

Competing interests: None

\section{Nozomi Okuno, Kazuo Hara, Nobumasa Mizuno, Susumu Hijioka, Hiroshi Imaoka, Kenji Yamao}

Department of Gastroenterology, Aichi Cancer Center Hospital, Nagoya, Japan

\section{References}

1 Yamao K, Sawaki A, Takahashi K et al. EUSguided choledochoduodenostomy for palliative biliary drainage in case of papillary obstruction: report of 2 cases. Gastrointest Endosc 2006; 64: 663-667

2 Itoi T, Sofuni A, Itokawa $F$ et al. Endoscopic ultrasonography-guided biliary drainage. J Hepatobiliary Pancreat Surg 2010; 105: 93-99

3 Savides TJ, Varadarajulu S, Palazzo L. EUS 2008 Working Group document: evaluation of EUS-guided hepaticogastrostomy. Gastrointest Endosc 2009; 69: 3-7

4 Martins FP, Rossini LG, Ferrari AP. Migration of a covered metallic stent following endoscopic ultrasound-guided hepaticogastrostomy: fatal complication. Endoscopy 2010; 42 (Suppl. 02): E126-127

5 Vila JJ. Initial experience with EUS-guided cholangiopancreatography for biliary and pancreatic duct drainage: a Spanish national survey. Gastrointest Endosc 2012; 76: $1133-1141$ Germany; Medico's Hirata Inc., Osaka, Japan). After contrast medium was injected, a 0.025-inch guide wire (VisiGlide; Olympus Medical Systems, Tokyo, Japan) was introduced through the needle and placed into the common bile duct. The fistula was dilated using a 9-Fr tapered biliary dilation catheter (Soehendra biliary dilation catheter; Cook Endoscopy; Bloomington, Indiana, USA). Finally, a fully covered metallic stent $(8 \mathrm{~mm} \times 8 \mathrm{~cm}$ Wallflex; Microvasive Endoscopy, Boston Scientific, Natick, Massachusetts, USA) was placed. Inward stent migration occurred immediately ( $\bullet$ Fig. 1), which would be a fatal complication $[4,5]$, and thereafter open surgical drainage was performed ( $\bullet$ Fig.2). Fortunately, the man was able to resume oral intake after surgery; however, 44 days
Bibliography

Dol http://dx.doi.org/

10.1055/s-0034-1392314

Endoscopy 2015; 47: E311

(c) Georg Thieme Verlag KG

Stuttgart · New York

ISSN 0013-726X

\section{Corresponding author}

\section{Kazuo Hara, MD}

Departments of Gastroenterology and Endoscopy Aichi Cancer Center Hospital

1-1 Kanokoden

Chikusa-Ku

Nagoya 464-8681

Japan

Fax: +81-52-7635233

khara@aichi-cc.jp 